\title{
Stochastic simulation of catalytic surface reactions in the fast diffusion limit
}

\author{
Ethan A. Mastny ${ }^{\text {a) }}$ \\ Department of Chemical and Biological Engineering, University of Wisconsin-Madison, \\ Wisconsin 53706-1607 \\ Eric L. Haseltine ${ }^{\text {b) }}$ \\ Division of Chemistry and Chemical Engineering, California Institute of Technology, 210-41, Pasadena, \\ California 91125 \\ James B. Rawlings ${ }^{\text {c) }}$ \\ Department of Chemical and Biological Engineering, University of Wisconsin-Madison, \\ Wisconsin 53706-1607
}

(Received 13 June 2006; accepted 13 October 2006; published online 21 November 2006)

\begin{abstract}
The master equation of a lattice gas reaction tracks the probability of visiting all spatial configurations. The large number of unique spatial configurations on a lattice renders master equation simulations infeasible for even small lattices. In this work, a reduced master equation is derived for the probability distribution of the coverages in the infinite diffusion limit. This derivation justifies the widely used assumption that the adlayer is in equilibrium for the current coverages and temperature when all reactants are highly mobile. Given the reduced master equation, two novel and efficient simulation methods of lattice gas reactions in the infinite diffusion limit are derived. The first method involves solving the reduced master equation directly for small lattices, which is intractable in configuration space. The second method involves reducing the master equation further in the large lattice limit to a set of differential equations that tracks only the species coverages. Solution of the reduced master equation and differential equations requires information that can be obtained through short, diffusion-only kinetic Monte Carlo simulation runs at each coverage. These simulations need to be run only once because the data can be stored and used for simulations with any set of kinetic parameters, gas-phase concentrations, and initial conditions. An idealized CO oxidation reaction mechanism with strong lateral interactions is used as an example system for demonstrating the reduced master equation and deterministic simulation techniques.
\end{abstract}

(C) 2006 American Institute of Physics. [DOI: 10.1063/1.2390696]

\section{INTRODUCTION}

Langmuir introduced modeling of gas adsorption on solid surfaces ${ }^{1}$ and his ideas were further developed by Hinshelwood $^{2}$ and Hougen and Watson. ${ }^{3}$ Their work provides methods for modeling chemical reaction networks that occur at gas-solid interfaces with ordinary differential equations. These models are easily expressed because they rely on the ideal surface assumption, which is that all molecules are randomly placed on the surface and feel the same environment, as in an ideal gas. Ideal surface methods provide models of macroscopic observables; however, they do not necessarily provide accurate models. ${ }^{4}$

Ideal surface methods ignore two important features of surface reactions: (1) reaction rates do not necessarily have the mass-action dependence that arises from random placement and (2) catalyst particle sizes may be not be large enough to assume the large number limit.

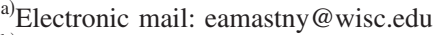

${ }^{b)}$ Electronic mail: haseltin@caltech.edu

${ }^{c)}$ Electronic mail: rawlings@engr.wisc.edu
}

\section{A. Mass-action dependence}

In the Langmuir-Hinshelwood-Hougen-Watson formalism a homogeneous species distribution is assumed, and elementary reaction rates are therefore proportional to reactant coverages. Lateral interactions between adsorbed species, surface heterogeneities, and bimolecular reactions may all create spatial correlations. ${ }^{5}$ Differential equation models have been developed that explicitly track pair correlations. ${ }^{6}$ As longer range patterns form on surfaces pair or higherorder correlation models lose accuracy. It has been shown experimentally that lateral interaction strength varies widely and can have substantial effect on system properties such as heats of adsorption, ordering of adlayers, and temperature desorption spectra. ${ }^{7}$ Systems that exhibit long-range correlations of surface species can be accurately modeled with kinetic Monte Carlo (KMC) simulations. KMC simulations track the locations of all the adsorbed molecules on a lattice surface as the molecules adsorb, desorb, react, and diffuse, and thus KMC simulations capture spatial correlations.

\section{B. Small particle size}

Chemically reacting systems are affected by fluctuations that arise from finite system sizes. These fluctuations have 
insignificant impact on chemical production rates for macroscopic catalyst particles, but can significantly change the behavior of the reaction network when small numbers of molecules are involved. It has been shown experimentally with field electron microscopy (FEM) that fluctuations induced by small molecule numbers (hundreds to thousands) can drive transitions between active and inactive reaction branches of $\mathrm{CO}$ oxidation. ${ }^{8} \mathrm{KMC}$ simulations of $\mathrm{CO}$ oxidation on nanoparticles have been used to show that internal fluctuations generate oscillatory behavior that does not occur in macroscopic models. ${ }^{9}$ Additionally, KMC simulations of $\mathrm{CO}$ oxidation have shown that the interplay between internal fluctuations and reaction network nonlinearity creates an optimal particle size. ${ }^{10}$ Many applications of heterogeneous catalysis employ small (10-100 $)$ supported catalyst particles. Therefore understanding small catalyst particle reaction behavior has useful applications.

\section{Surface reaction time scales}

In a review of applications of KMC simulations in heterogeneous catalysis Jansen and Lukkien stated, "The hardest part for a DMC [KMC] simulation is diffusion. The problem is that there are situations where the rate constant for diffusion is much larger than those for other reactions, and diffusion has no negative feedback that reduces the number of reactions/hops." 11 For example, experimental studies show that diffusion of $\mathrm{CO}$ on a $\mathrm{Pt}(111)$ surface at $300 \mathrm{~K}$ is greater than ten orders of magnitude faster than the $\mathrm{CO}-\mathrm{O}$ reaction event. ${ }^{12}$ Also on $\mathrm{Pt}(111)$ density-functional theory (DFT) simulations show that diffusion of oxygen atoms is eight orders of magnitude faster than the $\mathrm{CO}-\mathrm{O}$ reaction event. ${ }^{13}$

When this separation of time scales exists, KMC simulations of surfaces with realistic event rates are impractical for determining species production rates. During such a KMC simulation, the events chosen are mostly diffusion events (which have no direct effect on production rates) with rare reaction events.

Raimondeau and Vlachos reviewed methods for addressing this separation of time scales between diffusion and reaction events in KMC simulations. ${ }^{14}$ They proposed progressively increasing the diffusion rate until plateaus in the production rates of interest are reached. They state that if spatial patterns exist over small length scales, then the plateau is normally reached when diffusion rates are three to four orders of magnitude higher than reaction rates. Although this method is straightforward when applied to a system with a given surface coverage, the diffusion rate constants needed to reach the plateau may need to be modified as the surface coverage changes. Additionally simulating diffusion $10^{3}-10^{4}$ times more often than reaction is still an inefficient use of computational resources.

Many authors have used this "high but not too high diffusion rate" method described above to successfully study systems of practical interest. Liu and Evans studied temperature-programed reaction spectra and bifurcation diagrams of CO oxidation on $\mathrm{Pd}(100)$ with KMC simulations where $\mathrm{CO}$ diffusion rates were set to a value high enough to render the published results "insensitive" to the simulated diffusion rate. ${ }^{15}$ Völkening and Wintterlin studied the effect of adsorbed species interactions on the reaction order of $\mathrm{CO}$ oxidation on $\mathrm{Pt}(111)$ with both scanning tunneling microscopy and KMC simulations. ${ }^{16}$ They simulated fast CO diffusion events by implementing a $\mathrm{CO}$ hop for all $\mathrm{CO}$ molecules between every reaction event.

It is broadly assumed that when all reactants are highly mobile the adlayer is in equilibrium for the current coverages and temperature (see Zhdanov ${ }^{17}$ and Sundaresan and Kaza, ${ }^{18}$ for example). Starting from the assumption that the adlayer is in equilibrium several studies have tried to exploit their knowledge of the adlayer to enhance simulation speed. Makeev et al. used the equilibrated surface assumption along with the "microscopic time steppers" technique to simulate a lattice gas reaction in the high diffusion limit. ${ }^{19}$ In this work they assume that the coverages are the slow variables and all of the higher moments (pairs, triplets, etc.) are conditioned on the coverage. In our work we provide a derivation that shows that the coverages are indeed the slowly evolving variables in the fast diffusion limit. The computational strategy of Makeev et al. is to simulate coverage evolution which begins by equilibrating surfaces at a given coverage with a Metropolis Monte Carlo algorithm. They subsequently perform short KMC simulations without diffusion to determine the rate of change of coverages. They use the output from a collection of these short simulations to numerically estimate adsorbed species production rates. With this means of generating production rates they were able to construct a bifurcation diagram for an idealized $\mathrm{CO}$ oxidation mechanism with strong lateral interactions.

In a temperature programed desorption study of $\mathrm{CO}$ on $\mathrm{Rh}$ (100) Jansen described a method that attempts to sample an equilibrated surface while reducing the number of diffusion events needed for equilibration between reaction events. ${ }^{7}$ For a given configuration, the rate of diffusion is determined by evaluating the difference in energy of the final and initial states. This method of determining diffusion rates ensures that configurations are sampled with probabilities given by the Boltzmann weight. Unfortunately, this method requires choosing a preexponential factor that ensures species distribution equilibration between reaction events, similar to the method described by Raimondeau and Vlachos above.

Snyder et al. recently proposed a method called netevent KMC, which eliminates the disparity in time scales by treating rapid reversible events as a single net event. ${ }^{20}$ They proposed that the rate of this net event should be the absolute value of the difference between the forward and backward events. Simulation examples of well-mixed systems show that this method captures species number evolution correctly but suppresses the noise as a result of combining the forward and reverse processes. They applied this technique to a surface reaction problem with fast diffusion. Defining forward and reverse events required breaking the lattice up into $2 \times 2$ regions. However, it is not readily evident that netevent KMC can be applied to nonhomogeneous surfaces, which is the interest of this study. 


\section{Master equation methods}

KMC simulations are samples of a probability density whose evolution equation is the chemical master equation. ${ }^{21}$ For well-mixed chemical kinetics, the system states are all combinations of species numbers within the constraints of the mass balance and reaction network. As molecule numbers and numbers of species increase, the dimensionality of the master equation makes it difficult to solve for the probability of the full state.

Several authors have proposed methods for reducing the dimension of the master equation for spatially homogeneous systems. These order reductions are based on partitioning the master equation by knowledge of fast and slow reactions. These partitioning methods are used to justify simulation algorithms that follow the equilibrium, ${ }^{22,23}$ assumption. Haseltine and Rawlings recently proposed partitioning the master equation on the basis of fast and slow reactions as a means for reducing the order of the master equation. ${ }^{24}$ They outline a method that can be used to derive approximations for stochastic chemical kinetics. This method uses properties of conditional probability densities to derive approximate, Markovian master equations of a reduced set of variables. A similar method is applied in this work.

Nedea et al. developed a method for simulating a reactive system with infinitely fast diffusion in a one-dimensional pore, starting from the master equation. ${ }^{25}$ In this work they partition the master equation based on fast and slow reactions. Then by assuming that all configurations of a given order have the same probability, they derive expressions for the reaction propensities of the slow events. They then use these reaction propensities in KMC simulations that include only the slow events.

Chatterjee and Vlachos recently proposed a multiscale computational strategy for modeling surface reactions which couples fast processes (diffusion) on a small length scale with slow processes (adsorption and desorption) on the large length scale. ${ }^{26}$ They propose dividing the reaction surface into a grid of coarse cells. Each cell is characterized by its size and coverage. They propose calculating the reaction propensities of the slow reactions in the coarse cells using either mean field closures, quasichemical closures, or running short diffusion-only KMC simulations of equilibrated small lattices. Only slow reaction events are used to propagate the coarse cell states forward in time, thus allowing for long time steps. Their work provides a significant advancement in the ability to simulate a large coarse-grained lattice with fast diffusion rates.

In the infinite diffusion limit, researchers have proposed adiabatically eliminating diffusion events in systems that are not well mixed to leave only reaction events, thereby eliminating the spatial dependence. ${ }^{27}$ We propose performing a similar elimination for reaction-diffusion problems on lattices, which are unique because lattice sites can only be occupied by one species. In this paper, we use a partitioning scheme similar to Haseltine and Rawlings ${ }^{24}$ and Chatterjee and Vlachos ${ }^{26}$ to segregate between fast diffusion events and slow reaction events. This partitioning allows for the solution of a master equation with a reduced dimensional state (the species numbers). For the sake of brevity, many of the details of the derivation are omitted here; we refer the interested reader to an accompanying technical report for these details. ${ }^{28}$ We show that the reaction propensities in the reduced master equation can be obtained from short, diffusiononly KMC simulations. We provide details of what information is needed from the diffusion-only KMC simulations and how it should be used. We also show that this master equation reduces to a set of ordinary differential equations that close in the species coverages in the limit of a large lattice. We demonstrate these techniques with a CO oxidation model exhibiting strong lateral interactions.

\section{MASTER EQUATION ORDER REDUCTION}

\section{A. Notation}

For the balance of this paper a diffusion event is an event that changes the position of an absorbed species but does not change the overall number of each type of species on the lattice. A reaction event changes the number of some of the species on the lattice and can occur by adsorption/desorption or reaction.

The following variables describe the lattice size and species considered.

- $N_{s}$ is the number of sites on the lattice.

- $p$ is the number of chemical species.

The following variables describe different ways to represent lattice configurations and numbers of possible lattice configurations.

- $x$ is a state that represents a particular lattice configuration.

- $n$ is a vector of dimension $p$ containing the number of each species on the lattice.

- $\theta$ is a vector of dimension $p$ containing the coverage of each species on the lattice $\left(n / N_{s}\right)$.

- $n_{x}$ is a vector containing the species numbers in state $x$.

- $N_{x}$ is the number of possible $x$ states.

- $N_{n}$ is the number of possible $n$ states.

The following probability distributions characterize the lattice behavior. Although not explicitly written, all of the following probability densities are functions of time.

- $P_{x}(x)$ is the probability of having lattice configuration $x$.

- $P_{n, x}(n, x)$ is the joint probability of being in species number $n$ and lattice configuration $x$.

- $P_{n}(n)$ is the marginal probability of being in species number $n$.

- $P_{x \mid n}(x \mid n)$ is the conditional probability of being in configuration $x$ given species number $n$.

- $\hat{P}$ denotes an approximate probability distribution, in which the above subscript notations apply. 
TABLE I. Comparison of the dimension of $P_{x}$ and $P_{n}$ for different lattice sizes and species numbers.

\begin{tabular}{ccr}
\hline \hline Example & $N_{x}$ & \multicolumn{1}{c}{$N_{n}$} \\
\hline$N_{s}=4, p=1$ & 16 & 5 \\
$N_{s}=25, p=2$ & $10^{12}$ & 325 \\
$N_{s}=100, p=2$ & $10^{48}$ & 5050 \\
\hline \hline
\end{tabular}

The following expressions characterize transition rates from one state to another.

- $W_{\alpha, \beta}$ is the rate at which the system can move from lattice configuration $\beta$ to configuration $\alpha$, by a reaction or diffusion event.

- $K_{\alpha, \beta}$ is the rate at which the system can move from lattice configuration $\beta$ to configuration $\alpha$, by a reaction event.

- $D_{\alpha, \beta}$ is the rate at which the system can move from lattice configuration $\beta$ to configuration $\alpha$, by a diffusion event.

\section{B. Probability state dimension}

The difficulty that arises in solving the discrete master equation exactly is the dimension of the probability density. The dimension of the probability density is the number of accessible states or states that can be reached within the constraints of the mass balance and reaction mechanism. The probability density $P_{x}$ has the dimension of the number of possible lattice configurations $\left(N_{x}\right)$.

$$
N_{x}=(p+1)^{N_{s}} .
$$

The probability density $P_{n}$ has the dimension of the number of possible combinations of species numbers on the lattice $\left(N_{n}\right) . N_{n}$ is proportional to $N_{s}^{p}$. For a system with two species $N_{n}=\left(N_{s}+1\right)\left(N_{s}+2\right) / 2$.

Table I compares the dimensions of $P_{x}$ and $P_{n}$ for a few cases. The dimension of $P_{n}$ is significantly less than $P_{x}$ because the states in $P_{n}$ do not account for lattice positions of surface species. The numbers given in Table I demonstrate that, even for small lattices $\left(N_{s}=100\right)$, the probability density $P_{x}$ cannot be stored in a computer. This table also shows that for lattices with $O(100)$ sites, $P_{n}$ has a state dimension small enough to enumerate and evaluate. One purpose of this paper is to show that the model in $P_{x}$ can be accurately reduced to a model in $P_{n}$ when diffusion events are much faster than reaction events. This order reduction facilitates the use of the chemical master equation to solve for the probability density of the surface reaction for small lattices.

\section{Exact evolution of $n$}

The master equation of the full state is

$$
\frac{d P_{x}(x)}{d t}=\sum_{\beta} W_{x, \beta} P_{x}(\beta)-W_{\beta, x} P_{x}(x) .
$$

We define the marginal density $P_{n}(n)$ as

$$
P_{n}(n)=\sum_{x} P_{n, x}(n, x)=\sum_{\alpha} P_{x}(\alpha) \delta\left(n-n_{\alpha}\right),
$$

in which $\delta\left(n-n_{x}\right)$ is a function of value 1 when the coverage $n$ is the same as the coverage of configuration $x$ and zero otherwise. The evolution of $P_{n}$ is then

$$
\frac{d P_{n}(n)}{d t}=\sum_{\alpha} \frac{d P_{x}(\alpha)}{d t} \delta\left(n-n_{\alpha}\right)
$$

The summation in the full master equation (1) can be broken into separate summations over diffusion and reaction events, i.e., $W_{\alpha, \beta}=K_{\alpha, \beta}+D_{\alpha, \beta}$. Since diffusion events do not change species numbers on the lattice, summing over diffusion events in Eq. (1) does not affect $n$, and the resulting evolution equation for $P_{n}$ is

$$
\frac{d P_{n}(n)}{d t}=\sum_{\alpha, \beta}\left(K_{\alpha, \beta} P_{x}(\beta)-K_{\beta, \alpha} P_{x}(\alpha)\right) \delta\left(n-n_{\alpha}\right) .
$$

With some further manipulations we cast the right-hand side of Eq. (4) in terms of the joint density for later use. Let $n_{\alpha}$ $=\Delta n_{\alpha, \beta}+n_{\beta}$. Then

$$
\frac{d P_{n}(n)}{d t}=\sum_{\alpha, \beta} K_{\alpha, \beta} P_{n, x}\left(n-\Delta n_{\alpha, \beta}, \beta\right)-K_{\beta, \alpha} P_{n, x}(n, \alpha) .
$$

\section{Fast time scale}

Assumption 1. On the fast time scale the evolution of the joint density is affected by only diffusion events, i.e.,

$$
\begin{aligned}
\frac{d \hat{P}_{n, x}(n, x)}{d t} \triangleq & \sum_{\beta} D_{x, \beta} \hat{P}_{x}(\beta) \delta\left(n-n_{x}\right) \\
& -D_{\beta, x} \hat{P}_{x}(x) \delta\left(n-n_{x}\right) .
\end{aligned}
$$

From the arguments given in Sec. II C, $d \hat{P}_{n}(n) / d t=0$. The definition of the conditional density $\hat{P}_{x \mid n}(x \mid n)$ is

$$
\hat{P}_{x \mid n}(x \mid n)=\frac{\hat{P}_{n, x}(n, x)}{\hat{P}_{n}(n)} .
$$

By differentiating Eq. (7) and using Eq. (6), we derive the following evolution equation for the conditional density:

$$
\begin{aligned}
\frac{\delta \hat{P}_{x \mid n}(x \mid n)}{d t}= & \frac{1}{\hat{P}_{n}(n)} \sum_{\beta} D_{x, \beta} \hat{P}_{x}(\beta) \delta\left(n-n_{x}\right) \\
& -D_{\beta, x} \hat{P}_{x}(x) \delta\left(n-n_{x}\right) \\
= & \sum_{\beta} D_{x, \beta} \hat{P}_{x \mid n}(\beta \mid n)-D_{\beta, x} \hat{P}_{x \mid n}(x \mid n) .
\end{aligned}
$$

Equation (8) states that for a given $n$, the evolution of $x$ is Markovian and depends only on diffusion events. Remember that this equation holds only for fast time scales or a time scale during which no reaction events occur. Therefore, the KMC simulation that samples this master equation has a fixed $n$ and employs only diffusion events. Additionally, it can be shown that $\hat{P}_{n}(n)>0$ for all $0<t<\infty$, so the conditional density is well defined. 


\section{E. Approximate evolution of $\boldsymbol{n}$}

Assumption 2. The joint density of being in state $(n, x)$ can be approximated by the probability of being in state $n$ times the conditional probability of being in state $x \mid n$ from the fast time scale.

$$
P_{n, x}(n, x) \approx P_{n}(n) \hat{P}_{x \mid n}(x \mid n) .
$$

Substituting this assumption into the marginal density evolution, Eq. (5), gives

$$
\begin{aligned}
\frac{d P_{n}(n)}{d t}= & \sum_{\alpha, \beta} K_{\alpha, \beta} \hat{P}_{x \mid n}\left(\beta \mid n-\Delta n_{\alpha, \beta}\right) P_{n}\left(n-\Delta n_{\alpha, \beta}\right) \\
& -K_{\beta, \alpha} \hat{P}_{x \mid n}(\beta \mid n) P_{n}(n) .
\end{aligned}
$$

Equation (9) sums over all microscopic states in the marginal density. The consequence of this summation is more readily apparent by rewriting this equation in terms of reaction events; the details of this procedure are presented in an accompanying report. ${ }^{28}$ Then Eq. (9) is equivalent to

$$
\frac{d P_{n}(n)}{d t}=\sum_{j} k_{j}\left[\left\langle s_{j}\left(n-\nu_{j}\right)\right\rangle P_{n}\left(n-\nu_{j}\right)-\left\langle s_{j}(n)\right\rangle P_{n}(n)\right],
$$

in which $\left\langle s_{j}(n)\right\rangle=\Sigma_{\alpha} s_{j}(\alpha) P_{x \mid n}(\alpha \mid n)$ and $\nu_{j}$ is the change in $n$ due to reaction $j$ ( $\nu$ is an effective stoichiometric matrix). Thus, $\left\langle s_{j}(n)\right\rangle$ is the average number of $j$ reactions possible on the lattice given $n$. Additionally, Eq. (10) is Markovian. If the $\left\langle s_{j}(n)\right\rangle$ terms are known for all $n$, then the master equation for the marginal density can be solved exactly. These terms are a function of the distribution of microscopic states sampled for a given $n$.

\section{F. Equilibrium assumption and simulation strategy}

Assumption 3. On the slow time scale the $x$ states reach equilibrium due to diffusion before reaction events occur that change $n$.

$$
\frac{d \hat{P}_{x \mid n}(x \mid n)}{d t}=0
$$

Without appealing to the mean field or similar assumption, the $\left\langle s_{j}(n)\right\rangle$ terms in Eq. (10) are not available analytically. In the previous section, we defined $\left\langle s_{j}(n)\right\rangle$ as the expectation of the number of reaction $j$ from the probability density of the slow time scale. The master equation that governs the evolution of $P_{x \mid n}(\alpha \mid n)$ is Eq. (8). This master equation evolves with only diffusion events. Therefore, we can determine $\left\langle s_{j}(n)\right\rangle$ by running a KMC simulation with only diffusion events and coverage $n$. When this KMC simulation reaches equilibrium, as in Eq. (11), $s_{j}(n)$ can be measured over many sample times to determine $\left\langle s_{j}(n)\right\rangle$.

\section{G. Large lattice limit and deterministic evolution equations}

In the large lattice limit, the variables of interest are the fractional coverages of each species on the surface $\left(\theta_{i}\right)$ rather than species numbers. Instead of tracking the number of each type of reaction $s_{j}(n)$, we track $g_{j}(\theta)=s_{j}(n) / N_{s}$. When diffusion is much faster than reaction, the surface equilibrates between reaction events. Under equilibrated conditions, the results of equilibrium thermodynamics and statistical mechanics of the NVT ensemble apply to the surface. The theory of thermodynamics of small systems states that in the limit of large systems, observables such as $g_{j}(\theta)$ become intensive. In other words, $g_{j}(\theta)$ approaches a constant as the lattice size increases. ${ }^{29}$ Additionally, a classic result of statistical mechanics is that the probability distribution of an intensive variable becomes increasingly sharp as the system size increases. In the thermodynamic limit the probability distribution of this intensive variable is a delta function. ${ }^{30}$

In the derivation given in the accompanying technical report $^{28}$ it is shown that

$$
\frac{d\langle n\rangle}{d t}=\sum_{j} \nu_{j} k_{j}\left\langle s_{j}\right\rangle .
$$

When divided by $N_{s}$ Eq. (12) becomes

$$
\frac{d\langle\theta\rangle}{d t}=\sum_{j} \nu_{j} k_{j}\left\langle g_{j}\right\rangle
$$

A system initialized with a specific coverage $\theta$ samples an equilibrated probability distribution for each $g_{j}$. If the lattice is large, the probability distribution of each $g_{j}$ is a delta function. Therefore, $\left\langle g_{j}\right\rangle$ can be written as $g_{j}(\theta)$, and the right-hand side of Eq. (13) becomes deterministic,

$$
\frac{d \theta}{d t}=\sum_{j} \nu_{j} k_{j} g_{j}(\theta) .
$$

\section{EXAMPLE SYSTEM}

We demonstrate the usefulness of the master equation order reduction method using a simplified model of $\mathrm{CO}$ oxidation $\left(2 \mathrm{CO}+\mathrm{O}_{2} \rightarrow 2 \mathrm{CO}_{2}\right)$ occurring on a square lattice with periodic boundary conditions. This model consists of the following elementary steps and involves two types of adsorbed species, $\mathrm{CO}$ and $\mathrm{O}$,

$$
\begin{aligned}
& \mathrm{CO}(\mathrm{g})+*_{i} \rightarrow \mathrm{CO}_{i}, \\
& \mathrm{CO}_{i} \rightarrow \mathrm{CO}(\mathrm{g})+*_{i}, \\
& \mathrm{O}_{2}(\mathrm{~g})+*_{i}+*_{j} \rightarrow \mathrm{O}_{i}+\mathrm{O}_{j}, \\
& \mathrm{O}_{i}+\mathrm{O}_{j} \rightarrow \mathrm{O}_{2}(\mathrm{~g})+*_{i}+*_{j}, \\
& \mathrm{CO}_{i}+\mathrm{O}_{j} \rightarrow \mathrm{CO}_{2}(\mathrm{~g})+*_{i}+*_{j}, \\
& { }_{d_{1}} \mathrm{CO}_{i}+*_{j} \rightarrow *_{i}+\mathrm{CO}_{j},
\end{aligned}
$$


TABLE II. Reaction model parameter values.

\begin{tabular}{cccc}
\hline \hline Parameter & Value & Parameter & Value \\
\hline$\alpha$ & $1.6 \mathrm{~s}^{-1}$ & $\beta$ & Varies \\
$\gamma$ & $0.001 \mathrm{~s}^{-1}$ & $k_{r}$ & $1.0 \mathrm{~s}^{-1}$ \\
$\rho$ & $0.001 \mathrm{~s}^{-1}$ & $\varepsilon$ & $-2.0 \mathrm{kcal} / \mathrm{mol}$ \\
$T$ & $500 \mathrm{~K}$ & & \\
\hline \hline
\end{tabular}

$$
\mathrm{O}_{i}+*_{j} \stackrel{d_{2}}{\rightarrow} *_{i}+\mathrm{O}_{j}
$$

In this reaction mechanism, $i$ and $j$ are nearest-neighbor sites. We consider repulsive interactions of strength $\epsilon$ between adsorbed $\mathrm{CO}$ molecules on nearest-neighbor sites. The parameter values used in this paper are shown in Table II and are chosen to match the values used by Makeev et al. ${ }^{19}$ for this same system.

There are only five reaction events and two diffusion events shown in the mechanism. The number of events is expanded when the effects of interactions are included. There are five distinct $\mathrm{CO}$ desorption events, four distinct $\mathrm{CO}-\mathrm{O}$ reaction events, and four distinct $\mathrm{CO}$ diffusion events. The rate constants of these events are multiplied by $\exp (-\epsilon M / R T)$, where $M$ is the number of $\mathrm{CO}$ molecules neighboring the $\mathrm{CO}$ molecule involved in the reaction.

The primary reasons for choosing this reactive system to test our new methods are mechanistic simplicity and the fact that these repulsive interactions create long-range patterns on the surface. These patterns invalidate the mean field assumption. For example, Fig. 1 shows the difference between a random lattice and an equilibrated lattice with $\mathrm{CO}$ repulsive interactions. Using this CO oxidation mechanism with lateral interactions, we demonstrate how a surface with patterns can be modeled accurately. Additionally, Makeev et al. have studied the bifurcation diagram for this system with microscopic time steppers. Their results provide an accuracy check for our work.

\section{SIMULATION EXAMPLES}

\section{A. Small lattice Master equation solutions}

In Sec. II we showed that the full configurational master equation can be reduced to the coverage master equation in the fast diffusion limit. This reduced master equation has the form given by Eq. (10). In this equation the expected number of each reaction at a given coverage $\left\langle s_{j}(n)\right\rangle$ is unknown. The numerical value of $\left\langle s_{j}(n)\right\rangle$ can be found by running a diffusion-only KMC simulation in which only diffusion

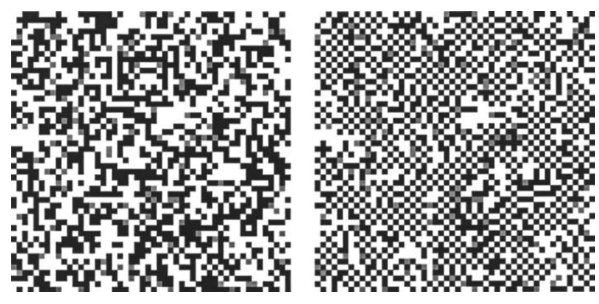

FIG. 1. Snapshots of a $50 \times 50$ lattice from a KMC simulation: CO (black), $\mathrm{O}$ (gray), and empty site (white). Left; random molecule placement and right; equilibrated. $\theta_{\mathrm{CO}}=0.45$ and $\theta_{\mathrm{O}}=0.04$.

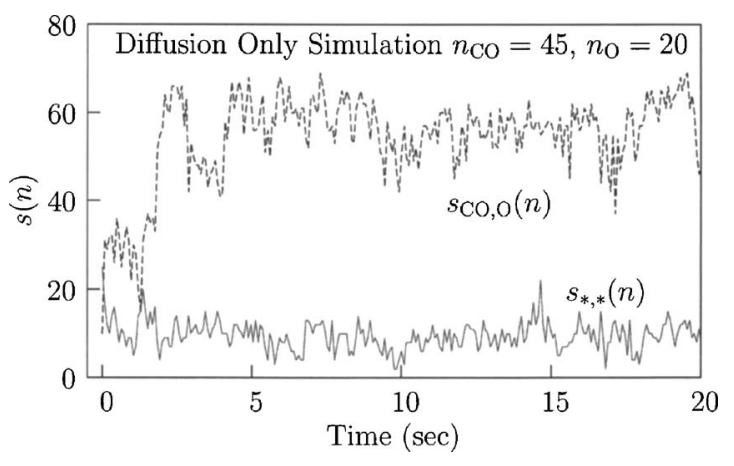

FIG. 2. Example of a diffusion-only simulation, where the numbers of two types of reactions are tracked. This is a $10 \times 10$ lattice, with $45 \mathrm{CO}$ and 20 oxygen molecules adsorbed. These data are used to determine the expected number of each reaction on the lattice for this particular coverage. This lattice was initialized with random molecule placement. To obtain a small confidence intervals for $\left\langle s_{j}(n)\right\rangle$ diffusion-only simulations are run much longer than shown here.

events occur at coverage $n$. This simulation samples the equilibrium distribution that occurs between reaction events at coverage $n$. During this simulation $s_{j}(n, t)$ is tracked for all of the possible reaction events. Figure 2 is an example of a diffusion-only KMC simulation. Given the $s_{j}(n, t)$ data from the KMC simulation, $\left\langle s_{j}(n)\right\rangle$ is calculated by finding the time average of $s_{j}(n, t)$.

To solve the master equation, $\left\langle s_{j}(n)\right\rangle$ must be available for all coverages. Table I shows that for our CO oxidation mechanism on a $5 \times 5$ lattice, there are 325 possible coverages and thus 325 diffusion-only KMC simulations must be run to determine $\left\langle s_{j}(n)\right\rangle$ for every $n$. Similarly 5050 diffusion-only KMC simulations must be run to determine $\left\langle s_{j}(n)\right\rangle$ for every $n$, on a $10 \times 10$ lattice. These simulations are short, need to be run only once, and can be distributed among many processors.

For this example, ten diffusion-only simulations were run at each $n$ for $2500 \mathrm{~s}$ of simulated time each. We found that this amount of simulation time was sufficient to ensure that the $95 \%$ confidence interval for $\left\langle s_{j}(n)\right\rangle$ was less than $2 \%$ of the calculated value of $\left\langle s_{j}(n)\right\rangle$.

Given the $\left\langle s_{j}(n)\right\rangle$ data from the KMC simulations and upon selecting a set of values for rate constants $k_{j}$, the righthand side of Eq. (10) is specified. Then, given an initial probability density of $P_{n}$, the linear differential equations, $d P_{n} / d t=A P_{n}$, can be solved to obtain $P_{n}$ at all times.

An important note is that the diffusion-only simulations need to be run only once. In this $\mathrm{CO}$ oxidation model the adsorption event rate constants $\alpha$ and $\beta$ are proportional to the gas-phase concentration of $\mathrm{CO}$ and $\mathrm{O}_{2}$, respectively. If the conditions above the surface change, the $A$ matrix of the master equation changes because of the effect on $k_{j}$, but the reaction rate constants have no effect on the values of $\left\langle s_{j}(n)\right\rangle$. Therefore, having run only one set of diffusion-only simulations, a master equation can be constructed and solved for any set of reaction event parameters.

When solving the master equation for a $5 \times 5$ lattice, an efficient way to calculate $P_{n}(t)$ is to calculate the matrix exponential, $P_{n}(t)=e^{A t} P_{n}(0)$. The dimension of the $A$ matrix for the $10 \times 10$ lattice is $5050 \times 5050$, however, thus making 


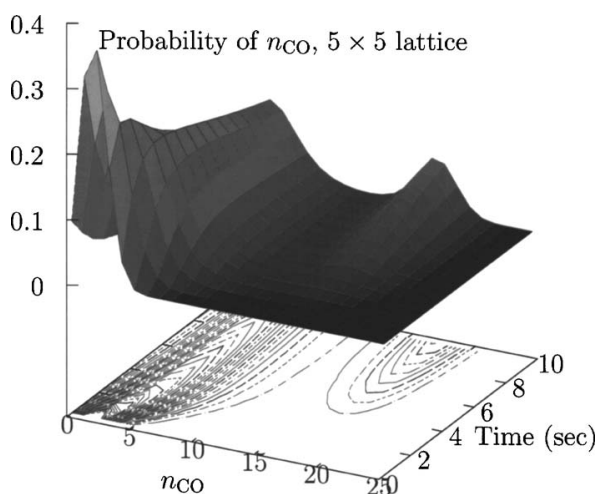

FIG. 3. Probability evolution of $\mathrm{CO}$ molecule number on a $5 \times 5$ lattice. Initial conditions: $n_{\mathrm{CO}}=2$ and $n_{\mathrm{O}}=20$, with $\beta=0.9$.

the matrix exponential time intensive to compute. Since this $A$ matrix has a sparsity of about $1 \%$, we used sparse matrixvector multiplication to evaluate the right-hand sides of the master equation. We then calculate $P_{n}(t)$ with a variable time step, explicit ordinary differential equation (ODE) solver.

In Figs. 3 and 4 an example solution for the master equation is shown for both the $5 \times 5$ and $10 \times 10$ lattices. In both cases, the initial coverages are $\theta_{\mathrm{CO}}=0.08$ and $\theta_{\mathrm{O}}=0.8$. In these figures the probability of the system having a specific $\mathrm{CO}$ coverage is plotted versus time. From these probability density surfaces, it is evident that the system is moving from low $\mathrm{CO}$ coverage to high $\mathrm{CO}$ coverage. It appears that the $5 \times 5$ lattice makes the transition more quickly than the 10 $\times 10$ lattice, meaning that species production rates depend on the lattice size.

These probability densities become stationary upon integrating the master equation for a sufficiently long time. Figures 5 and 6 show the steady-state probability densities of $n_{\mathrm{CO}}$ that correspond to the probability evolutions shown in Figs. 3 and 4 . Figure 5 shows that on a $5 \times 5$ lattice the surface has a significant probability to exist in either a low or high CO coverage state, although it is more likely to have a high coverage. This bimodal probability density denotes a surface that occasionally jumps between high and low $\mathrm{CO}$ coverages. On the $10 \times 10$ lattice, however, the low coverage peak has disappeared.

To check the accuracy of the simulation results generated by the reduced master equation we compared the sta-

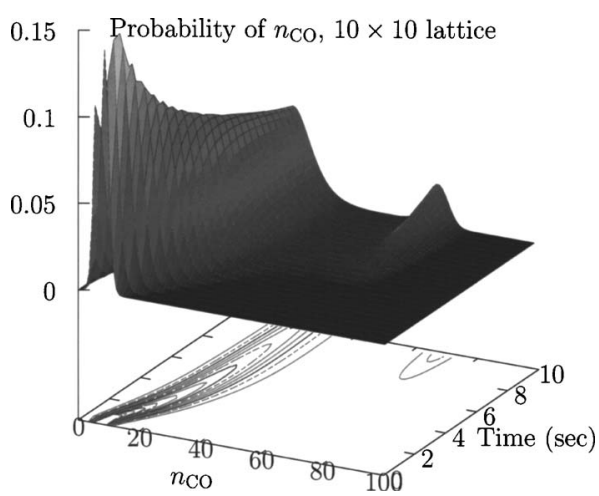

FIG. 4. Probability evolution of $\mathrm{CO}$ molecule number on a $10 \times 10$ lattice. Initial conditions: $n_{\mathrm{CO}}=8$ and $n_{\mathrm{O}}=80$, with $\beta=0.9$.

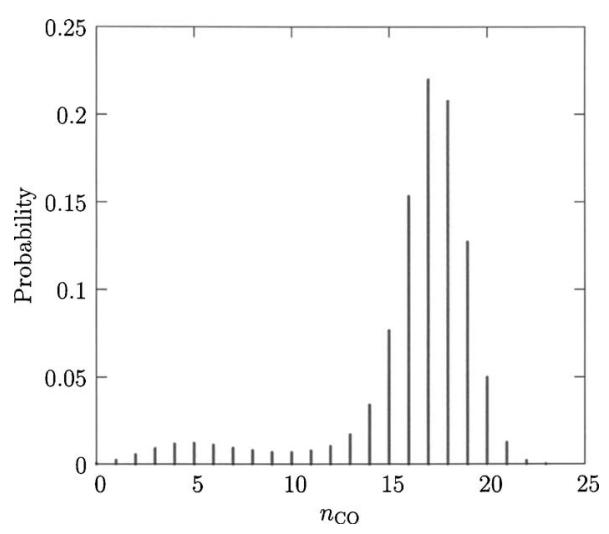

FIG. 5. Steady-state probability distribution of CO molecule number on a $5 \times 5$ lattice, with $\beta=0.9$.

tionary density of the $5 \times 5$ lattice, as shown in Fig. 5, to probability densities generated from KMC simulations with increasing diffusion rates. As diffusion rates increase the probability densities generated from the KMC simulations should converge to the density given by the reduced master equation. Figure 7 shows that this convergence occurs as expected. When $d=0.01 \mathrm{~s}^{-1}$ diffusion events occur with approximately the same frequency as reaction events. With every order of magnitude increase in the diffusion rate the ratio of diffusion events to reaction events increases by an order of magnitude. When $d=100$ and $1000 \mathrm{~s}^{-1}$ the expected numbers of diffusion events compared to reaction events are approximately $10^{4}$ and $10^{5}$, respectively. The computer time necessary to specify the right-hand side of the reduced master equation was about half the time needed to generate the probability distribution for $d=100 \mathrm{~s}^{-1}$. Besides giving the infinite diffusion limit, the advantage of the master equation is that it can be solved almost instantaneously for any set of kinetic parameters, while high diffusion rate KMC simulations would have to be repeated for any new set of kinetic parameters.

This master equation formulation is limited in a similar way that master equations are for well-mixed systems. The number of states grows rapidly as the number of species and lattice size increase. For example, a master equation for a $20 \times 20$ lattice with two species has 80601 states. A master equation for a $10 \times 10$ lattice with three species has 176851

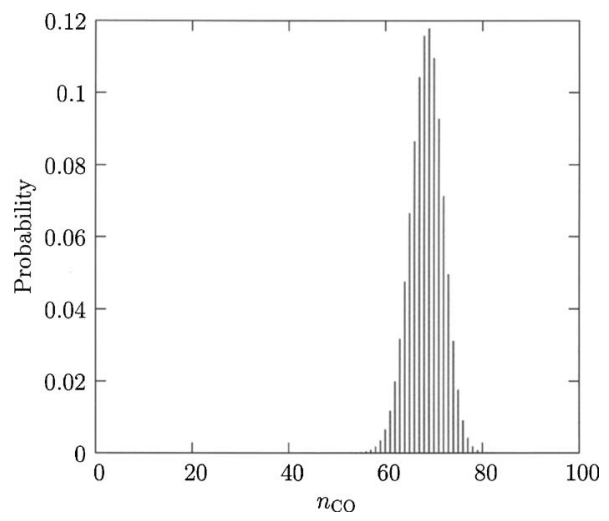

FIG. 6. Steady-state probability distribution of $\mathrm{CO}$ molecule number on a $10 \times 10$ lattice, with $\beta=0.9$. 


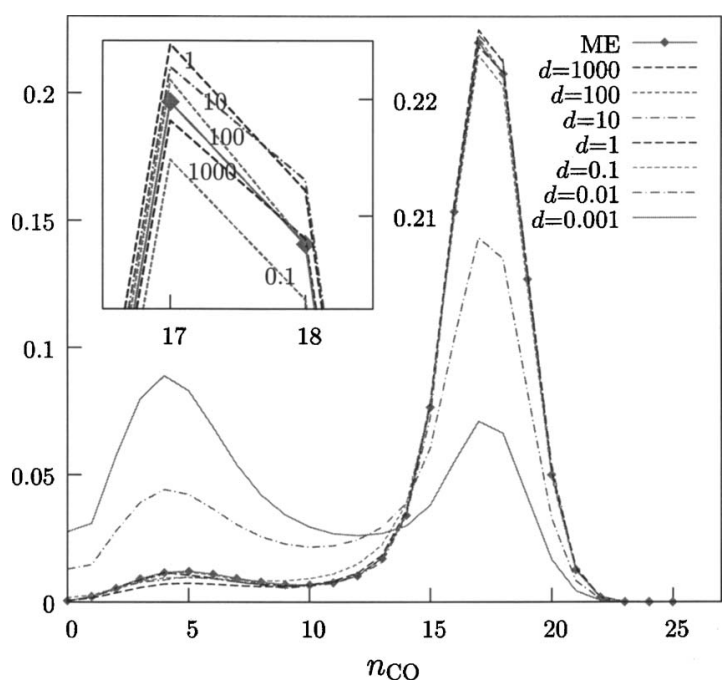

FIG. 7. Steady-state probability distributions of CO molecule number on a $5 \times 5$ lattice, with $\beta=0.9$. The line with points is the reduced master equation result; the other lines represent distributions from KMC simulations with different diffusion rates. The inset zooms in on the right peak and shows how the probability distributions converge to the reduced master equation distribution as diffusion increases.

states. We find that the limiting factor for solving the reduced master equation on the lattice is the number of diffusion-only simulations that must be run rather than calculating the solution of the differential equations.

\section{B. Large lattice: Deterministic differential equations}

In Sec. II G we showed that in the large lattice limit the master equation can be reduced to a set of differential equations in the coverages. These differential equations have the form

$$
\frac{d \theta}{d t}=\sum_{j} \nu_{j} k_{j} g_{j}(\theta) .
$$

An analytical functional form of $g_{j}(\theta)$ is unknown without the mean field assumption. For the small lattices, we used diffusion-only KMC simulations at every possible coverage to determine $s_{j}(n)$. For a large lattice, coverage is a continuous variable, thus making it impossible to simulate every possible coverage to determine $g_{j}(\theta)$. Instead we run diffusion simulations on a grid of coverage locations to find $g_{j}(\theta)$ at specific points and use interpolation between grid points.

The differential equations model the behavior of the large lattice limit; however, the diffusion-only KMC simula-

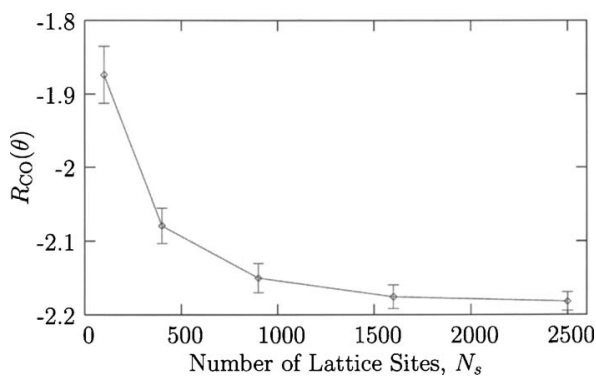

FIG. 8. Lattice size effects on $\mathrm{CO}$ coverage production rate, at $\theta_{\mathrm{CO}}=0.56$ and $\theta_{\mathrm{O}}=0.04$. At this coverage long-range patterns exist on the lattice.

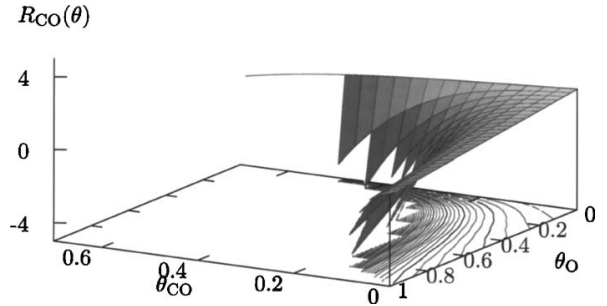

FIG. 9. The right-hand side of the mean field model $R_{\mathrm{CO}}(\theta)$ ODE at $\beta=2$.

tions used to determine $g_{j}(\theta)$ are calculated by simulations of a finite-size lattice. Therefore, a study of the finite-size effects must be conducted to determine if the diffusion-only simulation lattice is large enough to approximate the infinite lattice.

We studied the finite-size effects of this system by running a diffusion-only simulation at several lattice sizes $(10 \times 10,20 \times 20,30 \times 30,40 \times 40$, and $50 \times 50)$ with the same coverage. From these simulations we found $g_{j}(\theta)$ and calculated the coverage production rates $R(\theta)=\Sigma_{j} \nu_{j} k_{j} g_{j}(\theta)$. The production rate of adsorbed $\mathrm{CO}$ as a function of lattice size is shown in Fig. 8. Figure 8 shows that lattice size does affect the production rate. When plotted versus $1 / N_{s}$ the production rate changes linearly. This investigation shows that lattices of size of $40 \times 40$ or larger predict production rates that are good approximations to the infinite lattice production rates. At low CO coverage (little to no surface patterns) production rates are less dependent upon lattice size, and $30 \times 30$ is sufficient to approximate the infinite lattice.

Therefore, to determine the information for the righthand sides of Eq. (14), we ran diffusion-only simulations using a $40 \times 40$ lattice. The coverage space was divided into a grid of 22 coverages for each species, resulting in 231 simulations. From these simulation results, a lookup table for each type of reaction was created. Then $g_{j}$ can be found by lookup and interpolation for any $\theta$. With the lookup tables for $g_{j}(\theta)$, the differential equations for the coverages can be integrated, used for bifurcation analysis, optimization, design, or other system level tasks.

Figures 9 and 10 show the production rates of the mean field and patterned surface differential equations as a function of coverage, with a specific set of rate constants. The distinct shapes of the production rate surfaces highlight the differences between the mean field and reduced master equation models. We have also investigated the bifurcation diagram for this system where the oxygen adsorption rate con-

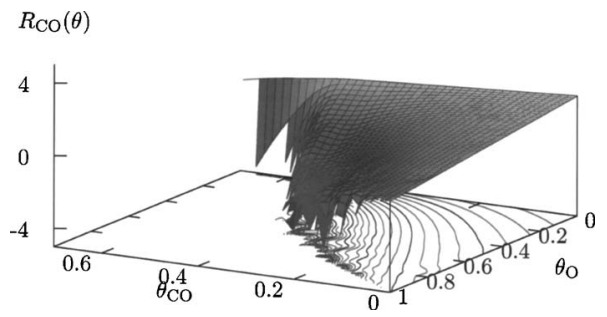

FIG. 10. The right-hand side of the reduced master equation model $R_{\mathrm{CO}}(\theta)$ $\mathrm{ODE}$ at $\beta=2$. 


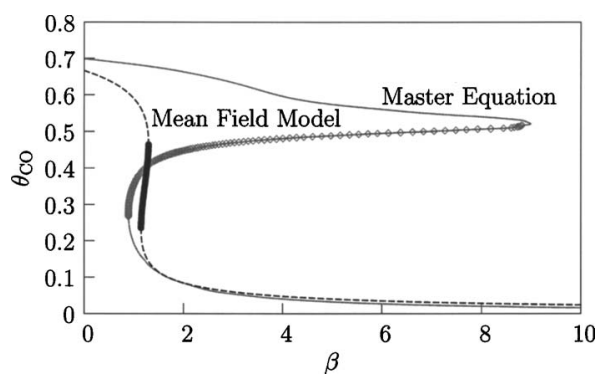

FIG. 11. The steady states of $\theta_{\mathrm{CO}}$ as a function oxygen gas concentration $(\beta)$ for both the reduced master equation and mean field models. Regions on the steady-state plots with symbols are unstable.

stant, $\beta$, is the bifurcation parameter. The steady states of $\mathrm{CO}$ coverage are shown for both the mean field and reduced master equation model in Fig. 11.

At high coverages of $\mathrm{CO}$, the $\mathrm{CO}$ molecules arrange themselves in a checkerboard pattern. The mean field model ignores all surface patterns leading to the significant modeling errors seen in Fig. 11 at high $\mathrm{CO}$ coverages. The checkerboard pattern makes if difficult for $\mathrm{O}_{2}$ to adsorb on the surface because there are no adjacent vacant sites. Only a large driving force, high $\beta$, can repopulate the surface with oxygen. The mean field model assumes a random orientation of all molecules and therefore predicts that there are a significant number of adjacent vacant sites for $\mathrm{O}_{2}$ adsorption even when $\mathrm{CO}$ coverage is high. The reduced master equation model steady-state curve closely matches the results given by Makeev et al. for the same system. ${ }^{19}$ These results also compare favorably with results from brute force KMC simulations run with high diffusion rates, as shown in Fig. 4 of Makeev et al.

\section{CONCLUSIONS}

In deterministic kinetics of well-mixed systems in which some reactions equilibrate much faster than others, the reaction equilibrium assumption is often used. The reaction equilibrium assumption is applied by solving for the equilibrium concentrations of species involved in the fast reactions subject to the constraints of the slow reactions. These equilibrated species concentrations are then used to propagate the slow reactions.

Many surface reactions have this same time-scale separation, in which diffusion events occur much faster than reaction events. This time-scale separation makes direct simulation of the surface reaction computationally intractable with KMC methods. We show how the stochastic master equation can be partitioned into fast diffusion events and slow reaction events. When we apply the reaction equilibrium assumption to this partitioned master equation, we produce a fast-time-scale master equation in which only diffusion events occur and a slow-time-scale master equation that tracks the probability density of only the species number states.

Scientists and engineers often want to track the species number density to predict chemical species production rates. Since the spatial states drop out of the slow-time-scale master equation, the dimension of this master equation is re- duced to a number that can be solved for small systems. In general, reaction propensities on the slow time scale are unknown. These reaction propensities can be determined by sampling the equilibrium distribution on the fast time scale with KMC simulations in which only diffusion events occur, subject to the slow reaction constraints (constant $n$ ). Once the reaction propensities are evaluated for all $n$ through KMC simulations, the slow-time-scale master equation is completely specified and can be solved.

This method allows us to solve for the probability density evolution of the species coverage on a surface, which is generally intractable because of the high dimension of the lattice master equation. It is also important that this solution does not assume a mean field (or other) species distribution, but rather accounts for the species distribution by sampling it on the fast time scale. This method has the same deficiencies as master equation solutions for any system: the dimension of the master equation increases rapidly with lattice size and species number. This method is only applicable to small lattices, however, the system size could be extended by solving the master equation only in the regions on sufficiently large probability as proposed by Munsky and Khammash. ${ }^{31}$

We have also shown that in the limit of large lattices, the reduced master equation reduces further to a set of ordinary differential equations that track the coverage of each adsorbed species. The rate of each reaction in the mechanism at a given coverage is again determined by sampling the equilibrium distribution in a KMC simulation. The data from these simulations can be stored for use in deterministic simulation with any kinetic parameters. If the number of species grows to such an extent that the whole coverage space cannot be simulated in advance, an adaptive tabulation method such as ISAT (Ref. 32) could be used to store the data needed to evaluate the differential equations.

One advantage of the methods proposed in this work over KMC simulation methods of surfaces is that sensitivities of the coverages with respect to reaction rate parameters $\left(k_{j}\right)$ are straightforward to evaluate from master equations and differential equations. In contrast, computing accurate sensitivities with $\mathrm{KMC}$ simulation is a computationally expensive process. Access to sensitivities is essential for performing system-level tasks efficiently, such as parameter estimation, design, and control. Calculating the sensitivities with respect to the interaction strength or temperature requires further diffusion-only KMC simulations, however, because these variables change the system properties on the fast time scale.

\section{ACKNOWLEDGMENTS}

This material is based upon work supported under a National Science Foundation Graduate Research Fellowship. The authors would like to thank Professor Yannis Kevrekidis and Professor Manos Mavrikakis for helpful discussions of aspects of this paper.

\footnotetext{
${ }^{1}$ I. Langmuir, J. Am. Chem. Soc. 40, 1361 (1918).

${ }^{2}$ C. N. Hinshelwood, The Kinetics of Chemical Change (Claredon, Oxford, 1940).
} 
${ }^{3}$ O. A. Hougen and K. M. Watson, Chemical Process Principles: Kinetics and Catalysis (Wiley, New York, 1947), Pt. 3.

${ }^{4}$ J. J. Carberry, in Chemical Reactor Theory: A Review, edited by L. Lapidus and N. R. Amundson (Prentice-Hall, Englewood Cliffs, NJ, 1977).

${ }^{5}$ H. C. Kang and W. H. Weinberg, Chem. Rev. (Washington, D.C.) 95, 667 (1995).

${ }^{6}$ S. Sundaresan and K. R. Kaza, Chem. Eng. Commun. 35, 1 (1985).

${ }^{7}$ A. P. J. Jansen, Phys. Rev. B 69, 035414 (2004).

${ }^{8}$ Y. Surchorski, J. Beben, E. W. James, J. W. Evans, and R. Imbihl, Phys. Rev. Lett. 82, 1907 (1999).

${ }^{9}$ E. Kurkina and N. Semendyaeva, Surf. Sci. 558, 122 (2004).

${ }^{10}$ Y. Gong, Z. Hou, and H. Xin, J. Phys. Chem. B 108, 17796 (2004).

${ }^{11}$ A. P. J. Jansen and J. J. Lukkien, Catal. Today 53, 259 (1999).

${ }^{12}$ R. Gomer, Rep. Prog. Phys. 53, 917 (1990).

${ }^{13} \mathrm{M}$. Mavrikakis (private communication).

${ }^{14}$ S. Raimondeau and D. G. Vlachos, Chem. Eng. J. 90, 3 (2002).

${ }^{15}$ D.-J. Liu and J. W. Evans, J. Chem. Phys. 124, 154705 (2006).

${ }^{16}$ S. Völkening and J. Wintterlin, J. Chem. Phys. 114, 6382 (2001).

${ }^{17}$ V. P. Zhdanov, Surf. Sci. 102, L35 (1981).

${ }^{18}$ S. Sundaresan and K. R. Kaza, Chem. Eng. Commun. 32, 333 (1985).

${ }^{19}$ A. G. Makeev, D. Maroudas, A. Z. Panagiotopoulos, and I. G. Kevreki- dis, J. Chem. Phys. 117, 8229 (2002).

${ }^{20}$ M. A. Snyder, A. Chatterjee, and D. G. Vlachos, Comput. Chem. Eng. 29, 701 (2005).

${ }^{21}$ D. T. Gillespie, J. Comput. Phys. 22, 403 (1976).

${ }^{22}$ Y. Cao, D. T. Gillespie, and L. R. Petzold, J. Chem. Phys. 122, 014116 (2005).

${ }^{23}$ C. V. Rao and A. P. Arkin, J. Chem. Phys. 118, 4999 (2003).

${ }^{24}$ E. L. Haseltine and J. B. Rawlings, J. Chem. Phys. 123, 164115 (2005).

${ }^{25}$ S. V. Nedea, A. P. Jansen, J. J. Lukkien, and P. A. J. Hilbers, Phys. Rev. E 67, 046707 (2003).

${ }^{26}$ A. Chatterjee and D. G. Vlachos, J. Chem. Phys. 124, 064110 (2006).

${ }^{27}$ M. A. Burschka, J. Stat. Phys. 45, 715 (1986).

${ }^{28}$ E. A. Mastny, E. L. Haseltine, and J. B. Rawlings, TWMCC, Technical Report No. 2006-02, 2006 (unpublished) (available at http:// www.che.utexas.edu/twmcc/).

${ }^{29}$ T. Hill, Thermodynamics of Small Systems Part I (Benjamin, New York, 1963).

${ }^{30}$ D. A. McQuarrie, Statistical Mechanics (Harper Collins, New York, 1973).

${ }^{31}$ B. Munsky and M. Khammash, J. Chem. Phys. 124, 044104 (2006).

${ }^{32}$ S. B. Pope, Combust. Theory Modell. 1, 41 (1997). 